\title{
Sensibilidade e Especificidade da Citologia Anal com Escova no Diagnóstico das Lesões Clínicas Provocadas pelo Papilomavírus Humano, Comparando Uma com Duas Coletas
}

\author{
Sensibility and Specifity of Anal Cytology with Cytobrush in the HPV Induced \\ Clinical Lesions Diagnosis, Comparing One to Two Smears
}

\author{
SIDNEY ROBERTO NADAL, TSBCP ${ }^{1}$; EDENILSONEDUARDOCALORE²; \\ CARMEN RUTH MANZIONE, TSBCP ${ }^{3}$; CIBELLE NUNES DEARRUDA ${ }^{4}$; JONATHAN DOYUN CHA ${ }^{4}$; \\ FERNANDABELLOTTIFORMIGA, ASBCP ${ }^{5}$; THIAGODA SILVEIRAMANZIONE, ASBCP ${ }^{5}$
}

\begin{abstract}
${ }^{1}$ Doutor pela Faculdade de Ciência Médicas da Santa Casa de São Paulo. Supervisor de Equipe Técnica de Proctologia do Instituto de Infectologia Emilio Ribas; ${ }^{2}$ Livre Docente pela Faculdade de Medicina da Universidade de São Paulo. Supervisor de Equipe Técnica de Proctologia do Instituto de Infectologia Emilio Ribas; ${ }^{3}$ Doutor pela Faculdade de Ciências Médicas da Santa Casa de São Paulo. Médica de Equipe Técnica de Proctologia do Instituto de Infectologia Emilio Ribas; ${ }^{4}$ Acadêmico da Liga de Proctologia da Faculdade de Ciências Médicas da Santa Casa de São Paulo; ${ }^{5}$ Médico Residente da Disciplina de Proctologia do Departamento de Cirurgia da Faculdade de Ciências Médicas da Santa Casa de São Paulo.
\end{abstract}

\begin{abstract}
NADAL SR; CALORE EE; MANZIONE CR; ARRUDA CN; CHA JD; FORMIGA FB; MANZIONE TS. Sensibilidade e Especificidade da Citologia Anal com Escova no Diagnóstico das Lesões Clínicas Provocadas pelo Papilomavírus Humano, Comparando Uma com Duas Coletas. Rev bras Coloproct, 2009;29(3): 297-302.

RESUMO: OBJETIVO: comparar os resultados da coleta única com duas amostras para avaliar se haverá melhora da sensibilidade e especificidade do exame. MÉTODO: Foram 112 doentes masculinos HIV-positivo com doença anal pregressa ou atual pelo Papilomavírus humano (HPV). As lesões HPV induzidas foram observadas em 58 deles. Colhemos material do canal anal utilizando duas escovas (cytobrush) Comparamos estatisticamente os resultados da primeira amostra com a soma das duas coletas. RESULTADOS: dos 58 doentes com lesões clínicas, a primeira amostra confirmou a doença em 40 (69\%) e a soma das duas coletas revelou lesões em $51(\mathbf{8 8} \%)$. Os resultados mostraram sensibilidade de $69 \%$ com a primeira coleta e $88 \%$ quando somadas as duas amostras. Essa diferença foi confirmada estatisticamente. A especificidade foi menor para as duas amostras, porém sem diferença estatística. CONCLUSÃO: Concluímos que a sensibilidade foi maior e a especificidade foi semelhante quando os resultados foram obtidos com a somação das duas amostras da citologia anal.
\end{abstract}

Descritores: Neoplasia intra-epitelial. Infecção pelo papilomavirus humano. Infecção pelo HIV. Carcinoma de células escamosas, canal anal.

\section{INTRODUÇÃO}

Com o surgimento da infecção pelo vírus da imunodeficiência humana (HIV), o carcinoma espinocelular (CEC) anal vem aumentando nos homens entre 30 e 40 anos de idade, especialmente nos praticantes do sexo anal receptivo, ${ }^{1}$ eparece estarintimamente relacionado com a infecção pelo papilomavírus humano (HPV). ${ }^{2,3}$

O HPV é responsável pelos condilomas acuminados e muitos deles contêm displasias. Vários são os fatores associados à maior probabilidade do desenvolvimento dessas displasias: a prática do sexo anal, a soropositividade para HIV,${ }^{4-7}$ a imunodepressão, ${ }^{8}$ as

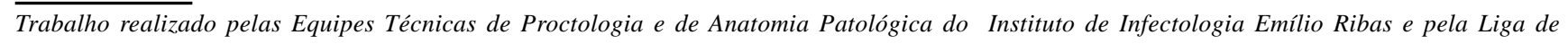
Coloproctologia da Faculdade de Ciências Médicas da Santa Casa de São Paulo. 
fases avançadas da infecção pelo HIV, ${ }^{9}$ as lesões verrucosas acima da linha pectínea,${ }^{6}$ as contagens de linfócitos T CD4 inferiores a 500/ $\mathrm{mm}^{3}{ }^{8,11-13}$ e o tipo viral do HPV..$^{14,15}$ Entretanto, o tempo de aparecimento dos condilomas, o tratamento tópico anterior e a associação com outras doenças sexualmente transmissíveis não parecem representar fatores de risco. ${ }^{6}$ Além disso, parece que o aspecto mais importante para o desenvolvimento do CEC anal é a duração da infecção pelo HIV. ${ }^{16}$

Essas displasias são atualmente conhecidas como neoplasias intra-epiteliais anais (NIA), que são as prováveis precursoras do tumor invasivo, com clara associação com os tipos de HPV de alto risco. ${ }^{6}$ Prova disso é a identificação do DNA do HPV nas células tumorais, que variou entre 35 e $90 \%$ nas diversas casuísticas publicadas. ${ }^{11-15,17}$

O risco de progressão pode estar ligado à severidade da NIA ${ }^{9,10}$ e, embora ainda não haja comprovação, o tratamento dessas lesões preveniria a evolução para carcinoma. ${ }^{6,8,9,11}$ NIA e câncer anal têm sido vistos com maior frequência na junção escamocolunar, na linha pectínea, que na margem anal (relação de 6:1), o que torna necessária a avaliação minuciosa dessa região.

Devido à possibilidade de detectar as lesões precursoras, programas padronizados para rastreamento do câncer anal, e dos seus precursores, e protocolos para tratamento da NIA deveriam ser instituídos. ${ }^{8}$ Sugere-se que a maioria dos casos de CEC anal tenha padrão biológico idêntico ao do câncer cervical. Da mesma forma que no colo do útero, o HPV no canal anal tem predileção pela junção escamocelular, parecendo lógico que esfregaços celulares colhidos dessa região possam auxiliar na detecção precoce das lesões citopáticas provocadas pelo vírus, selecionando os doentes para colposcopia anal, ou anuscopia ampliada, e biópsias.

Para candidatar técnicas para tratar as lesões precursoras, a padronização e a melhora dos métodos diagnósticos são essenciais, ${ }^{12,18}$ e acreditamos que a citologia anal possa servir para rastreamento, evitando a progressão para o carcinoma anal. ${ }^{19}$ Os vários trabalhos da literatura referiram sensibilidades para lesões subclínicas entre 45 e $98 \%,{ }^{14,20,21}$ talvez por falta de padronização quando à forma e local de coleta. Além disso, falhas de viés intra e interobservadores foram notadas na interpretação da NIA, ${ }^{7}$ colocando restrições ao método e justificando esses resultados variados. ${ }^{7}$ Também, não encontramos trabalhos sobre a efi- cácia do método para lesões clínicas do canal anal, o que seria importante, já que muitos profissionais não dispõem do anuscópio e nem sempre o toque retal é suficiente para sugerir sua presença.

Temos realizado citologia anal como rotina no seguimento dos doentes tratados de condilomas anais, com sensibilidade de $74 \%$ quando a escova foi introduzida até quatro centímetros a partir da margem anal. ${ }^{22}$ Nenhum deles referiu dor ou sangramento durante a coleta ou nos dias que se seguiram. No entanto, não há consenso na literatura sobre a real sensibilidade e especificidade da citologia na detecção da infecção pelo HPV, bem como há dúvidas sobre possíveis melhores resultados com a colheita de mais de uma amostra.

Assim sendo, idealizamos estudo de avaliação da citologia anal em doentes HIV-positivo portadores de infecção pelo HPV, com lesões pregressas ou presentes, utilizando duas coletas.

\section{OBJETIVO}

Avaliar a sensibilidade e a especificidade da citologia anal com escova para o diagnóstico de infecção clínica pelo HPV no canal anal de doentes HIVpositivo, comparando a coleta com uma segunda amostra retirada imediatamente após a primeira.

\section{MÉTODO}

Realizamos o estudo após avaliação e autorização da Comissão Científica e do Comitê de Ética em Pesquisa.

Os critérios de inclusão foram:

- homens adultos, com idade superior a 18 anos, portadores do HIV,

- com presença atual ou pregressa de condilomas no canal anal;

- que assinarem o termo de consentimento livre e esclarecido (TCLE)

Nesses doentes, após a assinatura do TCLE e antes de completar o exame proctológico com toque retal e anuscopia, faremos a coleta do material para citologia anal com escova apropriada (cytobrush), da seguinte maneira:

a) afastamento das nádegas para observar verrugas ou feridas anais na porção externa do canal anal.

b) introdução da escova fazendo movimentos suaves de rotação até o desaparecimento completo de 
suas cerdas evitando contato com as lesões externas, quando existentes.

c) da mesma maneira a retiramos e o material foi espalhado em lâminas de vidro acondicionados em frasco de plástico, cheio com álcool etílico a $70 \%$.

d) realizamos coleta da segunda amostra, seguindo a mesma técnica, utilizando outra escova e acondicionando a lâmina em outro frasco.

Os frascos foram enviados para o Laboratório de Anatomia Patológica para colorações pela hematoxilina-eosina e Pappanicolaou, e posterior leitura. O mesmo patologista avaliou todas as lâminas.

Como o objetivo foi saber se a segunda coleta aumentou a eficácia do exame na detecção das lesões induzidas pelo HPV no canal anal, a primeira coleta serviu como grupo-controle para somas dos resultados das duas amostras. Aplicamos os métodos apropriados para avaliação estatística dos resultados, utilizando o índice de 5\% para rejeição da hipótese de nulidade, e usamos a tabela $2 \times 2$, para conhecer a sensibilidade e a especificidade.

Alguns parâmetros são obtidos da Tabela $2 \times 2$. Os principais são:

- Sensibilidade: É a fração dos doentes que o teste é capaz de detectar. Matematicamente, $\mathbf{a} /(\mathbf{a}+\mathbf{c})$.

- Especificidade (ou Taxa de Verdadeiro Negativo): É a fração dos não-doentes que o teste é capaz de detectar. Matematicamente, $\mathbf{d} /(\mathbf{b}+\mathbf{d})$.

- Valor Preditivo Positivo: É a fração dos doentes com teste positivo que efetivamente tem a doença. Matematicamente, $\mathbf{a} /(\mathbf{a}+\mathbf{b})$.

- Valor Preditivo Negativo: É a fração dos doentes com teste negativo que efetivamente não tem a doença. Matematicamente, $\mathbf{d} /(\mathbf{c}+\mathbf{d})$.

Os resultados obtidos foram submetidos ao teste de comparação de duas amostras aceitando os resultados quando a probabilidade do acaso for menor ou igual a $5 \%$.

\section{RESULTADOS}

Colhemos amostras de 112 doentes masculinos com idades variando entre 18 e 62 anos e com média de 38 anos. Todos tinham história pregressa ou atual de verrugas anais. O procedimento foi realizado sem a necessidade de anestesia, devido não causar dor ou desconforto.

Durante o exame proctológico, incluindo anuscopia, identificamos lesões HPV induzidas no canal anal de 58 doentes. Neles, a primeira amostra foi positiva em $40(69 \%)$. Observamos a presença de NIA em outros 11 doentes cujo resultado da primeira coleta foi negativo, aumentando a eficácia para $88 \%$. A análise estatística $(\div$ quadrado) confirmou a diferença $(\mathrm{p}$ $=0,001)($ Tabela 1$)$

Utilizando a tabela $2 \times 2$, observamos que a sensibilidade para a primeira amostra foi de $68,96 \%$ e a especificidade foi de 53,76\%. Para as duas amostras, a sensibilidade foi $87,93 \%$ e a especificidade foi $42,74 \%$. Os valores preditivos positivos e negativos estão na Tabela 2. A avaliação estatística utilizando método de comparação de duas amostras mostrou diferença significativa para a sensibilidade o valor preditivo negativo. (Tabela 2)

Entre os 54 doentes cuja anuscopia não revelou lesão HPV induzida, a primeira amostra da citologia detectou NIA em $22(40,7 \%)$ e a segunda coleta mostrou-se positiva em mais sete. A análise estatística $(\div-$ quadrado) não mostrou diferença $(\mathrm{p}=0,247)$. Submetemos esses 29 doentes à colposcopia anal para biópsia dirigida e a lesão foi identificada e confirmada histologicamente em 22 (76\%).

\section{DISCUSSÃO}

Em diversos trabalhos, as escovas foram enfiadas no canal anal até a distância de dois, ${ }^{23}$ três, ${ }^{24,25} \mathrm{ou}$

Tabela 1 - Distribuição de 58 doentes HIV-positivo com doença clínica HPV induzida no canal anal, comparando os achados da citologia da primeira coleta com os das duas amostras. IIER, 2009.

\begin{tabular}{lllll}
\hline Lesão clínica HPV induzida & \multicolumn{2}{c}{ Citologia (+) } & \multicolumn{2}{c}{ Citologia (-) } \\
\cline { 2 - 5 } & $\mathbf{N}$ & \% & N & $\%$ \\
\hline $1^{\mathrm{a}}$ amostra & 40 & 69 & 18 & 31 \\
$1^{\mathrm{a}}+2^{\mathrm{a}}$ amostras & 51 & 88 & 07 & 12 \\
\hline
\end{tabular}

$p=0,0001$. 
Tabela 2 - Comparação dos parâmetros obtidos com a Tabela $2 \times 2$ nos achados da primeira coleta e das duas amostras de citologia anal de 112 doentes HIV-positivo. IIER, 2009.

\begin{tabular}{lccl}
\hline Parâmetros & primeira amostra(\%) & duas amostras (\%) & p \\
\hline Sensibilidade & 68,96 & 87,93 & $0,001 *$ \\
Especificidade & 53,76 & 42,74 & 0,125 \\
Valor preditivo positivo & 61,53 & 61,44 & 0,908 \\
Valor preditivo negativo & 61,75 & 75,86 & $0,033^{*}$ \\
\hline
\end{tabular}

(*) Análise da comparação de duas amostras - resultado com diferença significante.

quatro $^{26,27}$ centímetros $(\mathrm{cm})$ a partir da margem anal. Outros estudos relataram que o comprimento do canal anal variou entre 3,27 e 3,4 cm, em homens e entre 2,52 e 2,93 cm, nas mulheres. ${ }^{28-31}$ Esses dados justificaram os resultados que encontramos em pesquisa anterior, em que a eficácia da citologia anal foi superior quando as amostras foram obtidas até quatro $\mathrm{cm}$, quando comparadas às conseguidas em $2 \mathrm{~cm} .^{22}$

Em relação ao tipo de coleta, atualmente dispomos do método do Papanicolaou, em que as amostras são colhidas com escova (cytobrush) e o método da coleta úmida, o Thin Prep, em que a escova vem acondicionada em meio líquido e retorna a esse após seu uso. Estudos comparando ambos os métodos mostraram sensibilidade maior com o Thin Prep. ${ }^{32,33}$ No nosso estudo, aplicamos a escova seca por ser o meio disponível no Serviço.

O patologista avaliou a amostra, identificando a quantidade e a qualidade das células presentes (percentagem de células glandulares / transicionais) e classificando do mesmo modo que o esfregaço cervical, pelo Sistema Bethesda. ${ }^{34}$ Nesse sistema, os achados são distribuídos em: insatisfatório devido a celularidade deficiente; negativo para lesão intra-epitelial ou malignidade.(NIL), células escamosas atípicas de significado indeterminado (ASCUS), lesão intra-epitelial escamosa de baixo grau (LSIL) ou lesão intra-epitelial escamosa de alto grau (HSIL). ${ }^{35}$ No nosso estudo, optamos por usar a nomenclatura NIA, que tem correspondência histológica, com a SIL, sendo NIAA igual a HSIL e NIAB como LSIL.

Acreditamos que a citologia anal é bom método para rastreamento e seguimento, selecionando pacientes para colposcopia anal e biópsias, métodos mais caros, elaborados e invasivos. Sugerimos que pode ser usada por profissionais que não tenham anuscópios disponíveis, ou que realizem anuscopia, para diagnóstico de lesões HPV induzidas no canal anal, que muitas vezes não são detectadas ao toque retal. Todavia, a sensibilidade precisa ser melhorada, sendo necessárias mais pesquisas a esse respeito. Nesse ínterim, resolvemos pesquisar s sensibilidade com duas coletas, baseados na citação que a eficácia melhorou quando as citologias foram colhidas em intervalos de seis meses e os resultados foram somados. ${ }^{36}$ Não encontramos estudos semelhantes na literatura revista.

Consideramos que a melhora da sensibilidade de $69 \%$, com a primeira coleta, para $88 \%$, quando as duas amostras são consideradas, um alento para os portadores das lesões HPV induzidas e seus parceiros sexuais, no sentido de diagnosticar, com um método simples, as lesões precursoras do carcinoma anal. De qualquer forma, vamos aumentar a casuística e esperamos que o estudo seja repetido para confirmar o achado. Também, pretendemos avaliar a eficácia da Thin Prep, comparando-o ao método tradicional.

Observamos, também, que 59\% dos doentes sem lesões diagnosticadas ao exame proctológico completo apresentaram SIL à citologia. A colposcopia anal e o ácido acético não revelaram lesões no canal anal de $20 \%$ deles. Sugerimos, que a justificativa para essa observação seria o fato de se tratarem de doentes masculinos HIV-positivo, que têm elevado índice de infecção pelo HPV, sendo que muitos deles apresentavam lesões externas. Talvez, as células provenientes da doença clínica ou subclínica da margem anal pudessem contaminar o canal anal e ocasionar o resultado positivo. Também, suspeitamos que lesões existentes no interior das criptas anais, não detectadas pela colposcopia, poderiam ser reveladas pelo raspado. Concordamos com aqueles que sugeriram que, nos casos de citologia positiva com exame colposcópico discordante, elevada suspeição deve ser mantida e que 
nova avaliação seja feita em curto intervalo. ${ }^{37}$ De qualquer forma, quando a citologia revelar diagnóstico diferente da normalidade, a avaliação com o colposcópio deve ser realizada para permitir biópsias dirigidas.
Os resultados obtidos nas condições de execução do presente estudo permitiram concluir que a sensibilidade da citologia anal com duas coletas foi maior que com a coleta única e que a especificidade foi semelhante.

\begin{abstract}
OBJECTIVE: The aim of this study was to know if two smears may have better sensibility and specificity than a unique smear for anal cytology. METHOD:.There were 112 patients, males, HIV-positive, with current or previous anal HPVinduced lesions. Proctological examination revealed clinical disease in the anal canal of 58 of them. Smears were collect with cytobrushes. We compared results of the first smear to a sum of this with a second one. RESULTS: First smear was positive in 40 patients $(69 \%)$, and with both smears this incidence reached $88 \%$. Results showed sensibility of $69 \%$ to the unique smear, and $\mathbf{8 8 \%}$ when both were summed. Statistics revealed significant difference. Specificity was higher when both smears were summed, but statistics showed no difference. CONCLUSION: We concluded sensibility of anal cytology was better and specificity was similar when results were obtained with a sum of two smears.
\end{abstract}

Key words: Intra-epithelial neoplasia. Human papillomavirus infections. HIV infection. Squamous-cell carcinoma, anal canal.

\section{REFERÊNCIAS}

01. Lytwyn A, Salit IE, Raboud J, Chapman W, Darragh T, Winkler $B$ et al. Interobserver agreement in the interpretation of anal intraepithelial neoplasia. Cancer. 2005;103:1447-56.

02. Frisch M, Fenger C, van den Brule AJ, Sorensen P, Meijer CJ, Walboomers JM et al. Variants of squamous cell carcinoma of the anal canal and perianal skin and their relation to human papillomaviruses. Cancer Res. 1999;59:753-7.

03. Gervaz P, Allal AS, Villiger P, Buhler L, Morel P. Squamous cell carcinoma of the anus: another sexually transmitted disease. Swiss Med Wkly. 2003;133:353-9.

04. Palefsky JM, Holly EA, Gonzales J, Lamborn K, Hollander H. Natural history of anal cytologic abnormalities and papillomavirus infection among homosexual men with group IV HIV disease. J AIDS 1992;5:1258-65.

05. Carter PS, de Ruiter A, Whatrup C, Katzet DR, Ewings P, Mindel A et al. Human immunodeficiency virus infection and genital warts as risk factors for anal intraepithelial neoplasia in homosexual men. Br J Surg 1995;82:473-4.

06. Metcalf AM, Dean T. Risk of dysplasia in anal condyloma. Surgery 1995 ; 118:724-6.

07. Holly EA, Whittemore AS, Aston DA, Ahn DK, Nickoloff BJ, Kristiansen JJ. Anal cancer incidence: genital warts, anal fissure or fistula, hemorrhoids and smoking. J Natl Cancer Inst 1989; 81:1726-31.

08. Palefsky JM, Holly EA, Gonzales J, Lamborn K, Hollander H. Detection of human papillomavirus DNA in anal intraepithelial neoplasia and anal cancer. Cancer Res 1991; 51:1014-9.

09. Nadal SR, Manzione CR, Galvão VM, Salim VR, Speranzini MB. Perianal diseases in HIV-positive patients compared with a seronegative population. Dis Colon Rectum 1999;42:64954.

10. Manzione CR, Nadal SR, Calore EE. Oncogenicidade do papilomavírus humano e o grau de neoplasia intra-epitelial anal em doentes HIV positivo. Rev Assoc Med Bras 2004;50:282-285.

11. Critchlow CW, Surawicz CM, Holmes KK, Kuypers J, Daling JR, Hawes SE et al. Prospective study of high grade anal squamous intraepithelial neoplasia in a cohort of homosexual men: influence of HIV infection, immunosuppression and human papillomavirus infection. AIDS 1995;9: 1255-62.

12. Kiviat N, Rompalo A, Bowden R, Galloway D, Holmes KK, Corey $\mathrm{L}$ et al. Anal human papillomavirus infection among human immunodeficiency virus-seropositive and seronegative men. J Infect Dis 1990;162:358-61.

13. Levy AJ. Anal carcinoma. In: Levy AJ, HIV infection and development of cancer. Washington D.C., ASM Press, 1994.

14. Xi LF, Critchlow CW, Wheeler CM, Koutsky LA, Galloway DA, Kuypers J et al. Risk of anal carcinoma in situ in relation to human papillomavirus type 16 variants. Cancer Res 1998;58:3839-44.

15. Ryan DP, Campton CC, Mayer RJ. Carcinoma of the anal canal. N Engl J Med 2000;342:798-800.

16. Fagan SP, Bellows CF 3rd, Albo D, Rodriquez-Barradas M, Feanny M, Awad SS et al. Length of human immunodeficiency virus disease and not immune status is a risk factor for development of anal carcinoma. Am J Surg. 2005;190:732-5.

17. Palefsky J. Human papillomavirus and anal neoplasia. Curr HIV/AIDS Rep. 2008; 5:78-85.

18. Kreuter A, Brockmeyer NH, Hochdorfer B, Weissenborn SJ, Stucker M, Swoboda J et al. Clinical spectrum and virologic characteristics of anal intraepithelial neoplasia in HIV infection. J Am Acad Dermatol. 2005;52:603-8. 
19. Colquhoun P, Nogueras JJ, Dipasquale B, Petras R, Wexner $\mathrm{SD}$, Woodhouse $\mathrm{S}$. Interobserver and intraobserver bias exists in the interpretation of anal dysplasia. Dis Colon Rectum. 2003;46:1332-6.

20. Manzione CR, Nadal SR, Calore EE. Postoperative followup of anal condylomata acuminata in HIV-positive patients. Dis Colon Rectum 2003;46:1358-1365.

21. Mathews WC, Sitapati A, Caperna JC, Barber RE, Tugend A, Go U. Measurement Characteristics of Anal Cytology, Histopathology, and High-Resolution Anoscopic Visual Impression in an Anal Dysplasia Screening Program. J Acquir Immune Defic Syndr. 2004; 37:1610-1615.

22. Nadal SR, Calore EE, Nadal LRM, Horta, SHC, Manzione CR. Citologia anal para rastreamento de lesões préneoplásicas. Rev Assoc Med Bras 2007;53:147-151.

23. Papaconstantinou HT, Lee AJ, Simmang CL, Ashfaq R, Gokaslan ST, Sokol S et al. Screening methods for high-grade dysplasia in patients with anal condyloma. J Surg Res. 2005;127:8-13.

24. Vajdic CM, Anderson JS, Hillman RJ, Medley G, Grulich AE. Blind sampling is superior to anoscope guided sampling for screening for anal intraepithelial neoplasia. Sex Transm Infect. 2005;81:415-8.

25. Fox PA, Seet JE, Stebbing J, Francis N, Barton SE, Strauss S et al. The value of anal cytology and human papillomavirus typing in the detection of anal intraepithelial neoplasia: a review of cases from an anoscopy clinic. Sex Transm Infect. 2005;81:142-6.

26. Moscicki AB, Hills NK, Shiboski S, Darragh TM, Jay N, Powell $\mathrm{K}$ et al.. Risk factors for abnormal anal cytology in young heterosexual women. Cancer Epidemiol Biomarkers Prev. 1999;8:173-8.

27. da Costa e Silva IT, Gimenez FS, Guimarães RA, Camelo RT, Melo MND, Barros FS et al. Citologia anal como método de rastreamento para a detecção precoce do câncer anal: esfregaços com algodão hidrófilo são mesmo insatisfatórios? Acta Cir Bras. 2005;20:109-14.

28. Fenger $\mathrm{C}$. The anal transitional zone: location and extent. Acta Pathol Microbiol Scand [A]. 1979;87A:379-86.
29. Gold DM, Bartram CI, Halligan S, Humphries KN, Kamm MA, Kmiot WA. Three-dimensional endoanal sonography in assessing anal canal injury. Br J Surg.1999;86:365-70.

30. Regadas SM, Regadas FS, Rodrigues LV, Silva FR, Lima DM, Regadas-Filho FS. .Importância do ultra-som tridimensional na avaliação anorretal Arq Gastroenterol. 2005;42:226-32.

31. Jorge JM, Habr-Gama A. The value of sphincter asymmetry index in anal incontinence. Int J Colorectal Dis. 2000;15:30310.

32. Hutchinson ML, Zahniser DJ, Sherman ME, Herrero R, Alfaro $\mathrm{M}$, Bratti MC, et al. Utility of liquid-based cytology for cervical carcinoma screening: results of a population-based study conducted in a region of Costa Rica with a high incidence of cervical carcinoma. Cancer. 1999;87:48-55.

33. Lerma E, Quintana MJ, Quilez M, Esteva E, Carreras A, Bonfill $\mathrm{X}$, et al.. Effectiveness of liquid-based cytology and papanicolaou tests in a low risk population. Acta Cytol. 2007;51:399-406.

34. Coutinho JRH. Rastreamento de lesões pré-neoplásicas do ânus. Citologia anal e anuscopia de alta resolução novas armas para prevenção. Rev Col bras Cir, 2006:33:311-17.

35. Bethesda System 2001 Terminology [homepage on the Internet]. Bethesda (MD): National Cancer Institute; [cited 2005 Sep 29]. Available from: http://bethesda2001.cancer.gov/ terminology.html

36. Palefsky JM, Holly EA, Hogeboom CJ, Berry JM, Jay N, Darragh TM. Anal cytology as a screening tool for anal squamous intraepithelial lesions. J Acquir Immune Defic Syndr Hum Retrovirol. 1997;14:415-22.

37. Goldstone SE, Winkler B, Ufford LJ, Alt E, Palefsky JM. High prevalence of anal squamous intraepithelial lesions and squamous-cell carcinoma in men who have sex with men as seen in a surgical practice. Dis Colon Rectum. 2001; 44:690-8.

\section{Endereço para correspondência:} SIDNEY ROBERTO NADAL

Fone/Fax: (11) 3082-4942

E-mail: srnadal@terra.com.br

São Paulo, SP 